02

\title{
В каких случаях и почему стандартная модель туннелирующих двухуровневых систем неадекватно описывает низкотемпературную внутреннюю динамику реальных стекол
}

\author{
(C) Ю.Г. Вайнер ${ }^{1,2}$ \\ ${ }^{1}$ Институт спектроскопии РАН, \\ 108840 Москва, Троицк, Россия \\ ${ }^{2}$ Московский фризико-технический институт (Государственный университет), \\ 141701 Долгопрудный, Московская обл., Россия \\ e-mail: vainer@isan.troitsk.ru
}

Поступила в редакцию 25.02.2019 г.

В окончательной редакции 25.02.2019 г.

Принята к публикации 15.03.2019 г.

\begin{abstract}
Выполненные в последние годы с применением метода спектроскопии одиночных молекул эксперименты по изучению низкотемпературной динамики ряда молекулярных твердотельных сред с неупорядоченной внутренней структурой позволили получить новую информацию о динамике таких сред на локальном уровне. В одних веществах временно́е поведение большинства индивидуальных спектров одиночных флуоресцентных молекул, введенных в изучаемую среду в качестве локального спектрального зонда, соответствовало предсказаниям стандартной модели туннелирующих двухуровневых систем. В других поведение большинства спектров одиночных молекул имело более сложный характер, который трудно описать в рамках стандартной модели. Настоящая работа посвящена анализу результатов исследований низкотемпературной спектральной динамики одиночных флуоресцентных молекул в ряде неупорядоченных молекулярных систем (аморфный полиизобутилен, толуол, кумол, пропилен карбонат). Анализируется обнаруженная в экспериментах связь наблюдаемых отклонений от предсказаний теории с микроструктурой изучаемых систем и формой образца. Обсуждаются возможные причины отклонений наблюдаемой локальной спектральной динамики от предсказаний стандартной модели туннелирующих двухуровневых систем.
\end{abstract}

Ключевые слова: аморфные среды, стекла, спектроскопия одиночных молекул, туннелирующие двухуровневые системы, низкотемпературная динамика, флуоресцентные молекулы.

DOI: 10.21883/OS.2019.07.47930.108-19

\section{Введение}

Многочисленные эксперименты показывают, что при температурах ниже одного или нескольких кельвинов спектральные, термические, акустические и многие другие свойства большинства неупорядоченных твердотельных диэлектрических веществ, определяемые процессами внутренней динамики, очень похожи, мало зависят от физического строения и химического состава образца и сильно отличаются от соответствующих свойств высокоупорядоченных кристаллов (см., например, [1-5]). Это относится к неупорядоченным веществам самого разного вида: аморфные среды и стекла органической и неорганической природы, разнообразные полимеры и кристаллические среды, характеризующиеся наличием внутреннего беспорядка. Такие вещества часто называют просто стеклами. Для объяснения наблюдаемых при низких температурах динамических свойств стекол двумя группами теоретиков независимо была предложена простая теоретическая модель [6,7], которая объясняет наблюдаемую динамику инициированными акустическими фононами, туннельными переходами атомов, молекул или их групп между двумя минимумами на сложной потенциальной поверхности, ха- рактеризующей неупорядоченные твердотельные среды. По существу предложенная модель заменяет сложный потенциальный рельеф реальных стекол совокупностью невзаимодействующих двухъямных потенциалов, которые были названы туннелирующими двухуровневыми системами (ДУС). Невзирая на простоту, предложенная модель хорошо описывает большинство наблюдаемых при низких температурах динамических свойств стекол, и по этой причине была названа стандартной моделью туннелирующих ДУС, которую часто называют также стандартной моделью низкотемпературных стекол (CMH).

Выраженная универсальность низкотемпературных динамических свойств стекол самой разной природы, которые хорошо описываются в рамках одной модели, является одной из поразительных и загадочных особенностей твердых тел с неупорядоченной внутренней структурой. Несмотря на значительные усилия экспериментаторов и теоретиков, указанная универсальность до сих пор не нашла адекватной физической трактовки. Широко принято считать, что это обстоятельство является проявлением свойств структурного беспорядка в твердом теле. 
Несмотря на многочисленные усилия исследователей по изучению внутренней динамики стекол, ряд фундаментальных вопросов в этой области продолжает оставаться открытым. До сих пор неясно, какова физическая природа ДУС и насколько адекватно основанная на концепции ДУС стандартная модель описывает динамические свойства реальных стекол. Остается неясным, что является основной причиной наблюдаемой универсальности низкотемпературных свойств стекол, и каковы причины существования отклонений от предсказаний теоретической модели. Мы не знаем точного ответа на вопросы о том, до какой степени и в каких временныб́х и температурных интервалах динамические свойства стекол действительно идентичны и насколько адекватно они могут быть описаны в рамках единой теории. В последние годы интерес к низкотемпературной динамике неупорядоченных твердотельных систем и правомерности концепции ДУС значительно возрос. Это связано, в частности, с появлением новых твердотельных объектов, используемых при низких температурах, например, кубитов на сверхпроводящих структурах [8] или квантовых сверхпроводниковых однофотонных детекторов [9].

Одной из существенных причин наблюдаемой универсальности динамических свойств стекол при низких температурах является то обстоятельство, что большинство экспериментальных методов, используемых для исследования внутренней динамики таких сред, дают усредненные данные об изучаемых процессах. В подавляющем большинстве методов одновременно имеет место пространственное и временно́е усреднение. При изучении внутренней динамики по спектрам флуоресцентных молекул, вводимых в изучаемую среду в качестве спектральных зондов, большинство используемых для этих целей методов характеризуется также усреднением по ансамблю примесных молекул. Учитывая, что неупорядоченные среды обладают сложной пространственной микроструктурой с выраженным внутренним беспорядком, а также то, что характерные скорости динамических процессов в них могут простираться в широких временны́х пределах, указанные эффекты усреднения могут приводить к частичной или даже полной потере существенной части важной информации об индивидуальных особенностях протекания динамических процессов в различных неупорядоченных твердых телах.

Спектроскопия одиночных молекул (СОМ) является мощным оптическим методом изучения внутренней динамики диэлектрических твердотельных сред, который позволяет полностью устранить ансамблевое (и соответственно пространственное) усреднение получаемых данных о веществе и получить ценную информацию об изучаемых процессах на микроуровне (см., например, [10,11]). Индивидуальные спектры возбуждения люминесценции одиночных флуоресцентных молекул (ОМ), внедренных в прозрачную твердотельную матрицу в качестве спектрального нанозонда, содержат уникальные данные о динамике и других параметрах матрицы в ближайшем локальном окружении таких молекул. Отсутствие усреднения по ансамблю флуоресцентных молекул является принципиально важным преимуществом метода СОМ при изучении неупорядоченных твердотельных сред. Ширина индивидуальной бесфононной спектральной линии, наблюдаемой в спектре возбуждения люминесценции ОМ, дает информацию об ультрабыстрой динамике изучаемой матрицы в ее наноокружении, тогда как данные о частотах индивидуальных бесфононных линий и их изменениях содержат информацию о статических локальных параметрах окружающей среды и ее динамике в более медленной шкале времен.

Первые эксперименты с применением СОМ для изучения низкотемпературной динамики неупорядоченных твердых тел, выполненные в начале 90-х годов с аморфными телами и полукристаллическими молекулярными кристаллами при температурах жидкого гелия, наглядно продемонстрировали существование ДУС и показали, что на локальном уровне спектральная динамика сред, характеризующихся наличием локального беспорядка, отличается от соответствующей динамики, наблюдаемой в случае хорошо упорядоченных кристаллов [12,13]. Наблюдаемая динамика была объяснена взаимодействием электронных переходов ОМ, введенных в изучаемую матрицу, с медленными и быстрыми переходами в ДУС и интерпретирована как еще одно экспериментальное подтверждение существования ДУС в низкотемпературных стеклах. В работе [14], выполненной позже в 1999 г., были проведены более подробные исследования локальной спектральной динамики неупорядоченных твердотельных сред на примере двух неполярных полимеров: аморфного полиизобутилена (ПИБ) и полукристаллического полиэтилена, допированных флуоресцентными молекулами террилена. В этой работе впервые был применен метод регистрации временно́й эволюции бесфононных спектральных линий ОМ (спектральных траекторий), что позволило более детально исследовать локальную динамику изучаемой матрицы и сделало более наглядной интерпретацию полученных результатов. Спектральные траектории носили явно индивидуальный характер и демонстрировали случайные прыжки и дрейф частоты индивидуальных спектральных линий ОМ, а также спектральное расщепление. Часть из наблюдаемых траекторий демонстрировала временно́е поведение, хорошо согласующееся с предсказаниями $\mathrm{CMH}$, другая часть показывала поведение, выглядевшее как аномальное, которое трудно объяснить в рамках $\mathrm{CMH}$.

Позже в работах $[15,16]$ методика измерения спектров ОМ и их временно́го поведения была значительно усовершенствована путем перехода к параллельной регистрации большого числа флуоресцентных изображений ОМ с помощью высокочувствительной ПЗС-камеры. Обработка полученного массива данных при таком способе измерения позволяет получить информацию об индивидуальных спектрах ОМ и их временной эволюции для 
всей совокупности детектируемых с помощью CCD-камеры ОМ. Новая методика позволила существенно увеличить объем получаемой информации о спектральной динамике изучаемой среды и сделать ее более наглядной. С использованием указанной методики был выполнен цикл работ, имеющих целью более детальное изучение локальной спектральной динамики в ряде неупорядоченных твердотельных сред молекулярной природы [17-20]. Изучались аморфный ПИБ и низкомолекулярные стекла: толуол, кумол и пропилен карбонат. В качестве флуоресцентных нанозондов использовались молекулы тетратретбутилтеррилена и террилена. При исследованиях ПИБ измерялись спектры ОМ, находящихся как в толще, так и в сверхтонких приповерхностных слоях пленок из этого полимера. Была выявлена зависимость наблюдаемой локальной динамики от молекулярного веса полимера. Это проявлялось в виде резкого роста числа спектральных траекторий, поведение которых выглядело аномальным, несоответствующим предсказаниям СМH, при понижении величины молекулярного веса полимера. Было обнаружено также резкое увеличение числа аномальных траекторий в случаях ОМ, находящихся в сверхтонком приповерхностном слое полимера. а также при регистрации спектров ОМ, введенных в сверхтонкие полимерные пленки. Результаты измерения локальной спектральной динамики замороженных толуола, кумола и пропилен карбоната оказались интригующими. Большая часть наблюдаемых спектральных траекторий демонстрировала аномальное поведение, особенно сильно это проявлялось в случае пропилен карбоната.

Целью настоящей работы является анализ полученной в упомянутых экспериментальных исследованиях совокупности результатов с точки зрения применимости СМН для описания спектральной динамики ОМ в неупорядоченных молекулярных системах и обсуждения возможных причин ее отличий от предсказаний модели ДУС. Рассмотрение совокупности указанных результатов, несущих информацию о внутренней динамике изученных систем на локальном уровне, позволило сделать некоторые предположения о возможных причинах неприменимости СМН для описания динамических свойств молекулярных систем и поставить ряд вопросов, которые кажутся нам важными.

\section{Экспериментальные результаты}

В обсуждаемой работе [14] было измерено 70 спектральных траекторий в ПИБ и 14 в полиэтилене. Молекулярный вес полимеров не был указан. Наиболее важным результатом этой работы была регистрация траекторий, временно́е поведение которых выглядело аномальным, отличающимся от предсказаний СМН. Авторы разделили такие траектории на три типа. В первом случае (2 траектории) наблюдались прыжки спектральных траекторий ОМ между тремя частотными положениями. Указанное поведение авторы объяснили взаимо- действием наблюдаемой ОМ с трехуровневой системой, что явно не соответствует предсказаниям стандартной модели ДУС. Второй тип траекторий (13 траекторий) соответствовал более сложному поведению, которое авторы интерпретировали как результат взаимодействия наблюдаемой ОМ с взаимодействующими между собой ДУС. Так, например, в 4 случаях зарегистрированные спектральные траектории демонстрировали изменение относительной интенсивности спектрального дублета в ходе частотных прыжков, вызванных взаимодействием ОМ с двумя взаимодействующими между собой ДУС. Как отмечают авторы, указанное поведение также нельзя описать в рамках стандартной модели. Третий тип характеризовался непрерывным хаотическим дрейфом спектральной частоты ОМ (18 траекторий). Авторы заключили, что объяснить такое поведение в рамках СМН также очень трудно. В качестве одной из возможных причин было предложено рассмотреть высокую плотность ДУС.

В работах [17-20] изучались аморфный ПИБ с разным молекулярным весом $\left(M=3.9 \cdot 10^{2}, 2.5 \cdot 10^{3}, 3.38 \cdot 10^{4}\right.$ и $4.2 \cdot 10^{5} \mathrm{~g} / \mathrm{mol}$ ) и замороженные низкомолекулярные стекла: толуол, кумол и пропилен карбонат. Измерения осуществлялись при $T=4.5 \mathrm{~K}$, когда основной вклад в наблюдаемую динамику был обусловлен взаимодействием ОМ с окружающими ДУС (см., например, [16]), и при $T=7 \mathrm{~K}$, когда начинает проявляться взаимодействие с квазилокальными низкочастотными колебательными модами неупорядоченной твердотельной матрицы. Исследования ПИБ проводились в пленках различной толщины (в пределах 5-1100 nm) [17-19], а также в сверхтонких приповерхностных нанослоях пленки из этого полимера [20]. Толуол, кумол и пропилен карбонат вводились в промежуток между двумя покровными стеклами, толщина которого составляла несколько микрон (в атмосфере азота), и затем резко замораживались. В качестве флуоресцентных нанозондов использовались молекулы тетратретбутилтеррилена (ПИБ и толуол) и террилена (кумол и пропилен карбонат).

В ходе экспериментов с ПИБ, так же как и в работе [14], наблюдались спектральные траектории ОМ, согласующиеся с предсказаниями $\mathrm{CMH}$, и траектории, проявляющие аномальное поведение. Отношение числа спектральных траекторий, согласующихся с СМH, к числу аномальных траекторий зависело от молекулярного веса полимера и толщины полимерных пленок. В толстых пленках толщиной 1000-1100 nm, приготовленных из полимера с $M=4.2 \cdot 10^{5} \mathrm{~g} / \mathrm{mol}$, доля аномальных траекторий не превышала $10 \%$, что намного меньше, чем в работе [14], а при понижении молекулярного веса она резко увеличивалась. В случае пленок с $M=2.5 \cdot 10^{3}$ и $3.9 \cdot 10^{2} \mathrm{~g} / \mathrm{mol}$ подавляющее число спектральных траекторий демонстрировало поведение, не соответствующее предсказаниям СМН. На рис. 1 приведен ряд характерных спектральных траекторий, наблюдаемых в экспериментах с ПИБ с разным молекулярным весом. При уменьшении толщины пленок отно- 

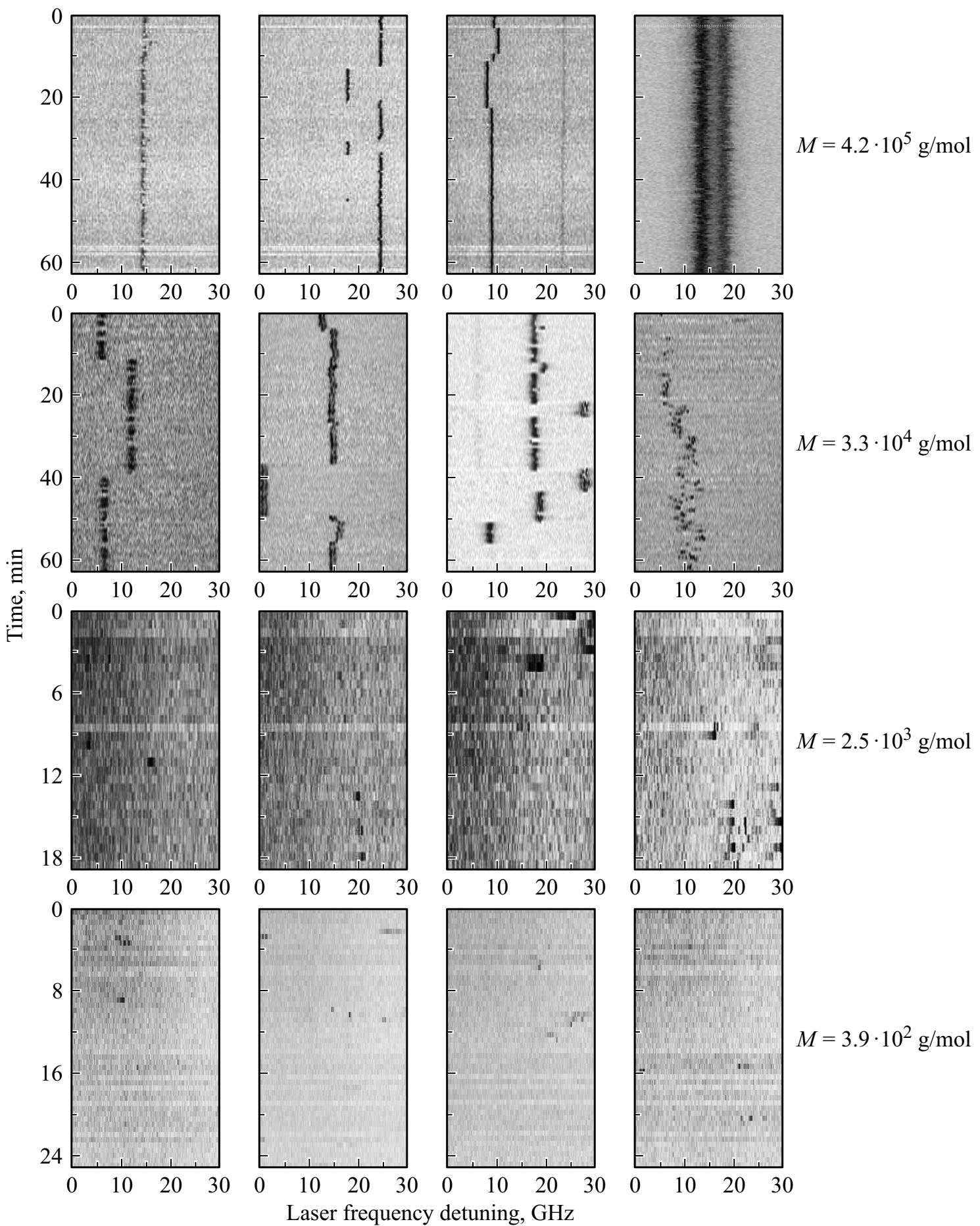

$M=2.5 \cdot 10^{3} \mathrm{~g} / \mathrm{mol}$

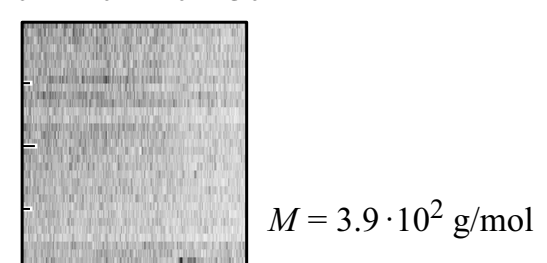

Рис. 1. Примеры спектральных траекторий, наблюдаемых при температуре $4.5 \mathrm{~K}$ в пленках из полиизобутилена с разным молекулярным весом, допированных флуоресцентными молекулами тетратретбутилтеррилена.

сительное число аномальных спектральных траекторий возрастало (до 50\% у пленок толщиной 4-5 nm). Было обнаружено также, что число аномальных траекторий возрастает при увеличении мощности возбуждающего ОМ лазерного излучения. При увеличении мощности с 0.8 до $47 \mathrm{~W} / \mathrm{cm}^{2}$ относительное число аномальных траекторий возрастало более чем в два раза.
Измерения спектральных траекторий ОМ, введенных в сверхтонкие приповерхностные слои пленок из ПИБ с $M=4.2 \cdot 10^{5} \mathrm{~g} / \mathrm{mol}$, толщиной $\sim 500 \mathrm{~nm}$, показали, что на расстояниях менее $20 \mathrm{~nm}$ от поверхности пленки спектральная динамика детектируемых ОМ начинает заметно отличаться от соответствующей динамики молекул в толще полимера. При приближении к поверхно- 

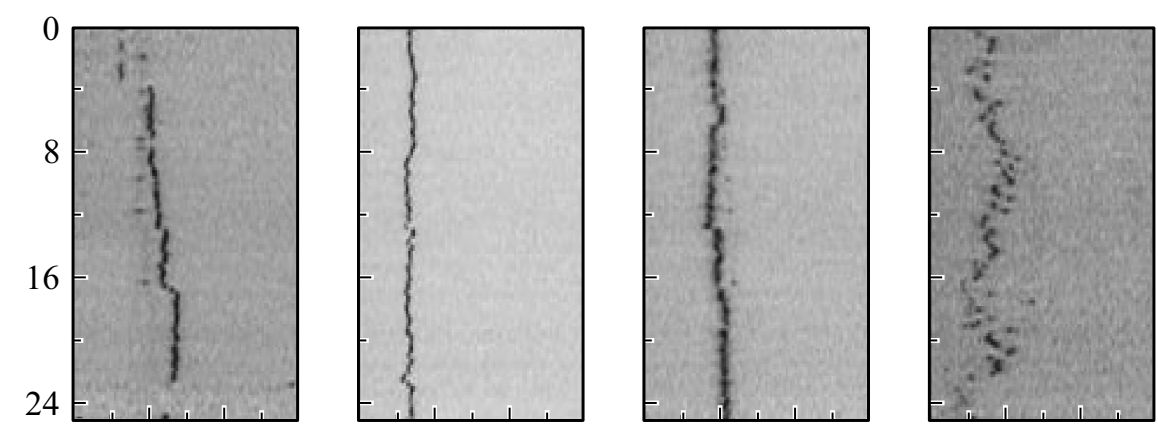

Toluene
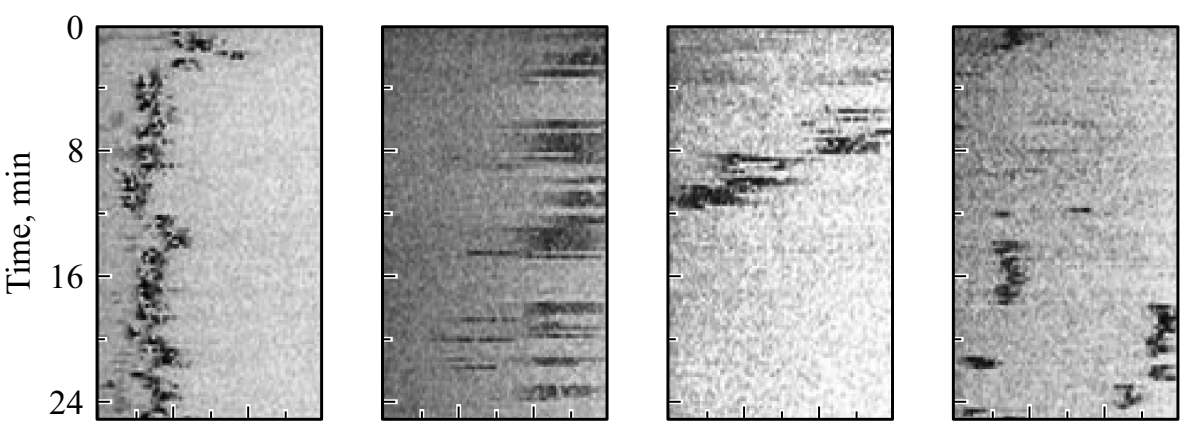

Cumene
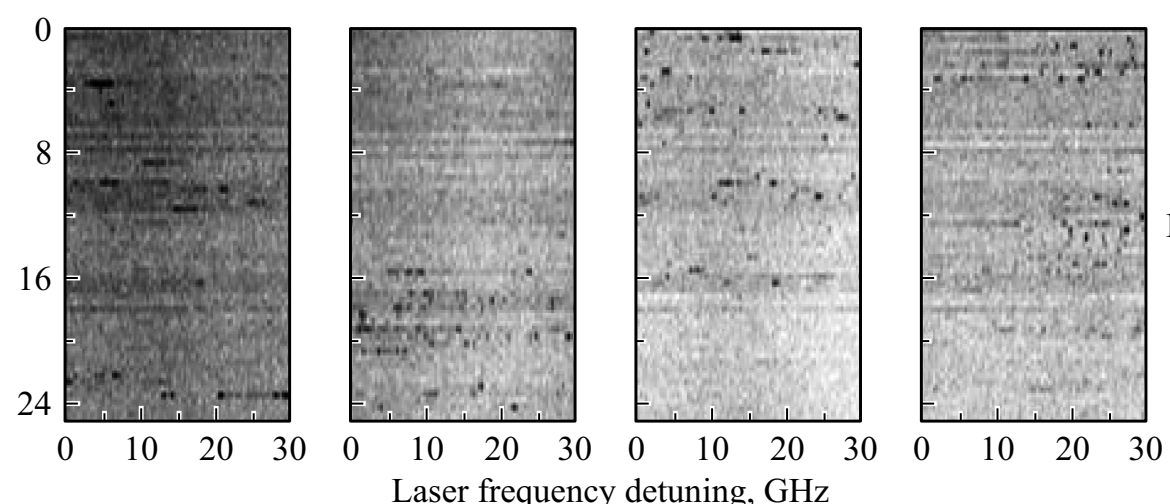

Propylene carbonate

Laser frequency detuning, $\mathrm{GHz}$

Рис. 2. Примеры спектральных траекторий, наблюдаемых в низкомолекулярных стеклах при температуре 4.5 К. Сверху вниз: толуол, кумол и пропилен карбонат.

сти спектральные траектории все большего числа ОМ (до $\sim 50 \%$ ) демонстрировали аномальное поведение.

Эксперименты с низкомолекулярными стеклами показали резкое отличие наблюдаемой в них локальной спектральной динамики от динамики, предсказываемой СМН. В большом числе случаев, помимо прыжков частоты и расщепления спектральных линий, которые можно приписать взаимодействию с ближайшими ДУС, измеренные спектральные траектории демонстрировали также невоспроизводимые хаотические дрейфы и прыжки частоты. Особенно сильное отличие в поведении наблюдаемых спектральных траекторий наблюдалось при исследованиях пропиленкарбоната. В этом случае практически все траектории демонстрировали совершенно нерегулярное, хаотическое временно́е поведение. Оптические сигналы от ОМ появлялись внезапно на некой случайной частоте, на короткое время и быстро исчезали. На рис. 2 приведены характерные примеры спектральных траекторий, регистрируемых при изучении низкомолекулярных стекол.

\section{Анализ результатов и обсуждение}

Представляется, что наиболее важным результатом, следующим из полученных в обсуждаемых работах экспериментальных данных, является тот экспериментальный факт, что на локальном уровне во всех изученных веществах наблюдались процессы спектральной динамики, проявляющие аномальный характер, который было трудно, а в ряде случаев невозможно, объяснить в рамках стандартной модели туннелирующих ДУС. Особенно большой интерес вызывает наблюдение в высокомолекулярном ПИБ спектральных траекторий ОМ, временно́е поведение которых нельзя объяснить в рамках СМН. Полиизобутилен известен как аморфный полимер. Его низкотемпературные свойства, определяемые процесса- 
ми внутренней динамики, хорошо описываются в рамках СМН. Поэтому факт обнаружения в этом веществе процессов локальной динамики, не подчиняющихся предсказаниям стандартной теории низкотемпературных стекол, является очень важным и имеет принципиальное, с точки зрения нашего понимания свойств неупорядоченных твердотельных веществ, значение. Он однозначно говорит о возможности нарушений предсказаний $\mathrm{CMH}$ на локальном уровне, даже в том случае, когда на макроуровне динамические свойства неупорядоченного вещества хорошо описываются в рамках $\mathrm{CMH}$.

В связи с этим фактом возникает вопрос: не является ли хорошо известная универсальность СМН для описания внутренней динамики широкого круга неупорядоченных твердотельных сред результатом сильного усреднения локальных параметров динамических процессов, протекающих в таких средах? Действительно, эффекты пространственного и временно́го усреднения, присущие традиционным экспериментальным методам, могут сильно сглаживать локальные особенности динамических процессов в таких средах. В результате отличия реальных свойств исследуемых неупорядоченных веществ на локальном уровне от предсказаний стандартной модели могут сильно маскироваться и не проявляться при измерениях. В случае ПИБ с высоким молекулярным весом, когда относительное число аномальных траекторий, не согласующихся с предсказаниями СМН, невелико, вполне можно предположить, что указанные эффекты усреднения являются основной причиной того факта, что на макроуровне этот аморфный полимер при низких температурах проявляет свойства, которые находятся в хорошем соответствии с предсказаниями СМН.

Следующим интересным и важным результатом, полученным в рассматриваемых работах, представляется тот экспериментальный факт, что в случае замороженных толуола, кумола и пропилен карбоната бо́льшая часть наблюдаемых траекторий имела аномальный характер. В случае толуола относительное число аномальных траекторий составляло чуть более половины, в случае кумола намного меньше, а в случае пропилен карбоната практически все наблюдаемые траектории проявляли ярко выраженный аномальный характер. К сожалению, полученной экспериментальной информации недостаточно для того, чтобы адекватно объяснить физическую причину наблюдаемого поведения.

В случае низкомолекулярных стекол, особенно в случае пропилен карбоната, совершенно не очевидно, что эффекты усреднения могут полностью замаскировать резко выраженное, хаотическое временно́е и спектральное поведение траекторий ОМ, которое наблюдается в этих средах. В связи с этим возникает следующий, не менее принципиальный вопрос: почему столь нерегулярное и сложное поведение спектров ОМ, отражающее сложную локальную динамику окружения этих молекул, на макроскопическом уровне достаточно хорошо описывается в рамках $\mathrm{CMH}$ ? В частности, хорошо известно, что быстрозамороженный толуол проявляет на макроуровне типичные свойства стекла. Этот непростой вопрос также остается открытым. Для ответа на него нужны более детальные данные о внутренней динамике рассматриваемых веществ, полученные на локальном уровне. В зависимости от этих данных можно будет понять, действительно ли и каким образом в ходе усреднения те или иные детали протекания локальных динамических процессов в этих веществах маскируются и не проявляются в измерениях.

Хорошо известно, что в большинстве случаев развитые теории хорошо описывают идеальные объекты, например идеальный кристалл, а в случае реальных объектов, в которых, как правило, имеются те или иные отличия от предположений модели, теории дают лишь приближенное описание объекта. Поэтому вопрос заключается не столько в том факте, что рассматриваемые эксперименты показали наличие аномальных спектральных траекторий в изученных веществах, а в том, каков их вклад в наблюдаемые свойства вещества в целом? Если этот вклад значителен, теория будет описывать такие вещества неадекватно и необходимо разрабатывать более совершенные модели. Если же такой вклад незначителен и мало влияет на точность описания объекта, острой необходимости в совершенствовании теории нет. Указанный вопрос тесно примыкает к широко обсуждаемым в научном сообществе вопросам: нуждается ли в настоящее время СМН в существенной доработке, в каких случаях она применима и насколько точно описывает наблюдаемые явления? Перечисленные вопросы приобретают в последнее время все большее внимание, в частности, в связи с развитием таких новых областей, как нанофизика и нанотехнологии. В этих областях изучается и разрабатывается большое число новых сверхмалых объектов, свойства которых сильно зависят от деталей их локальной структуры, так как эффективного усреднения по объему в таких случаях нет. В частности, изучение и применение таких объектов при криогенных температурах требует разработки более адекватных моделей для описания их динамических свойств при низких температурах (см., например, [21]). Поэтому факт экспериментального наблюдения в ряде неупорядоченных твердотельных молекулярных систем спектральных траекторий, демонстрирующих во многих точках макроскопически однородного образца явно выраженное отличие локальной динамики от предсказаний теории, указывает на актуальную необходимость разработки новых, более детальных теоретических моделей для описания низкотемпературной динамики неупорядоченных твердотельных сред. Такие работы сейчас ведутся (см., например, [9,21]).

Рассмотрим теперь важный вопрос о возможных физических причинах наблюдаемых отличий временно́го поведения спектральных траекторий ОМ от предсказаний СМН. Авторы работы [14] выдвинули два предположения: высокую локальную плотность ДУС и взаимодействие между ними. Возможное взаимодействие между ДУС и необходимость его учета в теории низкотемпературных стекол давно обсуждается в литературе (см., 
например, [23] и ссылки там). Этот эффект обусловлен электродипольным взаимодействием между ДУС и их взаимодействием с участием акустических фононов. Он становится заметным лишь при очень малых расстояниях между ДУС и по этой причине может проявляться лишь при высокой локальной плотности ДУС. Поэтому вопросы учета возможного взаимодействия между ДУС и рассмотрения высокой их плотности взаимно связаны: с повышением плотности ДУС характерное расстояние между ними возрастает и возрастает их взаимодействие.

Имеющихся экспериментальных данных недостаточно для адекватного ответа на вопросы о физической природе наблюдаемой в анализируемых работах аномальной локальной динамики. Это может быть результатом высокой плотности ДУС в исследуемых веществах и сильным взаимодействием между ними. Возможно также, что такие вещества характеризуются низкой величиной потенциальных барьеров. Еще одной возможной причиной может быть большая величина взаимодействия между ОМ и ДУС в указанных системах. Нельзя исключить, что причиной аномального поведения части спектральных траекторий, особенно в случае пропилен карбоната, являются локальные низкотемпературные структурные релаксации или существенно более быстрый характер протекающих в этом веществе динамических процессов по сравнению с временнобй шкалой экспериментов. Отмеченное непонимание физической природы локальной аномальной динамики, наблюдаемой в рассмотренных экспериментах, говорит о том, что продолжение экспериментальных и теоретических исследований низкотемпературной динамики неупорядоченных твердотельных тел продолжает быть актуальным. Этот вывод подтверждается, в частности, появлением все новых работ в этой области, небольшая часть которых упоминается в настоящей работе.

Интригующим результатом, полученным в работе [17] при исследованиях ПИБ, является явно выраженная зависимость локальной динамики от молекулярного веса полимера. Несмотря на небольшой, со статистической точки зрения, объем полученных в этих исследованиях данных, зависимость наблюдаемой динамики от молекулярного веса полимера не вызывает сомнений. Имеющихся данных, к сожалению, и в этом случае совершенно недостаточно для того, чтобы прояснить физические причины этой зависимости. Это может быть возрастающая роль концов полимерных цепочек при понижении молекулярного веса, когда их плотность возрастает, или возрастание подвижности самих цепочек, когда их длина уменьшается. Вне зависимости от ответа на этот вопрос полученный результат указывает на то, что на локальном уровне наблюдаемая динамика твердотельных молекулярных систем может сильно зависеть от внутренней структуры вещества. Этот важный вывод подтверждается и другими результатами, полученными в рассматриваемых работах. Так, например, еще одним интересным экспериментальным результатом, полученным в работе [20], является тот факт, что локальная спектральная динамика флуоресцентных ОМ в сверхтонких приповерхностных слоях ПИБ толщиной менее $20 \mathrm{~nm}$ сильно зависит от расстояния от этих молекул до поверхности. Более того, обнаруженная зависимость позволяет, хотя бы грубо, объяснить наблюдающееся в работе [19] при изучении пленок из высокомолекулярного ПИБ возрастание относительного числа аномальных спектральных траекторий при уменьшении толщины пленок возрастанием относительного числа OM, находящихся вблизи поверхности полимера, при уменьшении толщины пленки. Из обнаруженного факта, что большинство наблюдаемых в высокомолекулярном ПИБ аномальных траекторий соответствует ОМ, находящимся в приповерхностном слое пленок из этого полимера, следует важный вывод: локальная динамика в толще этого полимера очень хорошо описывается в рамках стандартной модели ДУС. Этот вывод имеет принципиальное для науки о динамике неупорядоченных твердотельных сред значение: найдена твердотельная неупорядоченная система (ПИБ с высоким молекулярным весом), динамические свойства которой хорошо описываются в рамках стандартной модели низкотемператуных стекол как на макро-, так и на микроуровне.

Свойства и структура поверхности и приповерхностных слоев твердотельных тел трудно поддаются изучению и плохо поняты, особенно в случае таких сложных объектов как полимеры. Поэтому ответить на вопрос, какова физическая природа обнаруженной зависимости, полученные в ходе проведенных исследований данные не позволяют. Авторы работы [20] лишь перечислили ряд возможных физических причин наблюдавшейся зависимости. Тем не менее говорить об отличии микроструктуры вещества в приповерхностных слоях образца из такого „мягкого“ материала, как ПИБ, от его микроструктуры в толще образца кажется вполне приемлемым даже в случае макроскопически однородного материала Поэтому вполне можно рассматривать обнаруженные отличия временно́го поведения спектральных траекторий ОМ в сверхтонком приповерхностном слое указанного полимера как еще одно экспериментальное подтверждение зависимости низкотемпературной динамики неупорядоченных твердотельных молекулярных систем от их внутренней микроструктуры. Эффект отличия параметров внутренней динамики в тонких приповерхностных слоях образца из неупорядоченного твердотельного вещества от их значений в толще образца, обнаруженный в работе [20], имеет большое значение в нанофизике и нанотехнологиях, где роль поверхности и приповерхностных слоев нанообъектов играет огромную, а во многих случаях превалирующую роль.

Все результаты, полученные в рассматриваемых работах, были получены в результате анализа временно́го поведения спектральных траекторий ОМ, внедренных в изучаемое вещество. Интерпретация спектральных траекторий, которые демонстрируют временно́е поведение, хорошо согласующееся с предсказаниями $\mathrm{CMH}$, 
достаточно очевидна и не вызывает каких-либо принципиальных вопросов. А как корректно интерпретировать траектории, временно́е поведение которых выглядит аномальным, тем более что они имеют разный характер? Это, например, случаи, когда помимо воспроизводимых прыжков частоты спектральных линий ОМ между двумя или четырьмя положениями (в общем случае, между $2^{n}$ положениями, где $n-$ число ближайших ДУС, с которыми взаимодействует ОМ) и спектрального расщепления наблюдаемые траектории демонстрируют хаотический дрейф частоты и нерегулярные, невоспроизводимые спектральные прыжки между случайными положениями. Другой случай, когда мы наблюдаем воспроизводимые прыжки частоты спектральных линий ОМ между 3 или 5 положениями. В отдельную группу следует выделить ОМ, демонстрирующие явную зависимость параметров какой-либо траектории от параметров другой. Если в последних двух случаях мы с большой вероятностью можем предполагать, что наблюдаемые траектории демонстрируют поведение, не согласующееся с предсказаниями СМН, то делать однозначный вывод о том, что аномальные спектральные траектории первого типа не могут быть описаны в рамках СМ без должного обоснования представляется совершенно неправильным. Так, например, можно предположить, что кажущееся аномальным поведение наблюдаемых спектральных траекторий ОМ может быть вполне объяснено высокой плотностью ДУС в изучаемой среде.

С целью проверки справедливости такого предположения для объяснения наблюдаемого в работе [20] аномального поведения спектральных траекторий в тонком приповерхностном слое пленок из высокомолекулярного ПИБ высокой плотностью ДУС в таком слое авторами этой работы были выполнены модельные расчеты спектральных траекторий ОМ для разных плотностей ДУС. Для этого в рамках СМН моделировалось временно́е поведение спектральных траекторий ОМ при различных плотностях ДУС с использованием значения константы связи хромофор-ДУС и параметров ДУС, взятых из работы [22], в которой было показано, что такие параметры хорошо описывают индивидуальные спектры ОМ в близких молекулярным системах.

Расчеты показали, что повышение плотности ДУС приводит в основном к увеличению спектральной ширины моделируемых траекторий и бо́льшим флуктуациям их средней частоты. Траектории, характеризующиеся сильным дрейфом или невоспроизводимыми хаотическими прыжками частоты, аналогичные аномальным траекториям, наблюдавшихся экспериментально, в этих расчетах получить не удалось. Поэтому основной вывод, который был сделан авторами указанной работы по результатам модельных расчетов, гласил, что наблюдаемые в эксперименте аномальные спектральные траектории ОМ, не согласующиеся с предсказаниями СМ, не могут быть объяснены повышенной плотностью ДУС в приповерхностных слоях полимера. Полученные в этой работе результаты очень интересны, но, к сожалению, не проясняют многие детали. Это связано, по нашему мнению, с тем, что в рассмотренных расчетах не учтено, что при повышении локальной плотности ДУС одновременно резко возрастает вероятность взаимодействия между ними. Поэтому основной вывод этой работы, по нашему мнению, следовало бы изложить в следующей форме: „Наблюдаемые в эксперименте аномальные спектральные траектории ОМ, не согласующиеся с предсказаниями СМ, не могут быть объяснены одним лишь повышением плотности ДУС в приповерхностных слоях полимера“.

\section{Заключение}

1. Низкотемпературная внутренняя динамика неупорядоченных молекулярных систем при наблюдении на локальном уровне имеет более сложный характер, чем в случае измерений на макроуровне, и не проявляет в той же степени универсальное поведение, которое наблюдается при измерениях традиционными методами, дающими сильно усредненные данные. В частности, локальная динамика таких сред может сильно зависеть от микроструктуры вещества.

2. Стандартная модель низкотемпературных стекол, основанная на концепции невзаимодействующих туннелирующих двухуровневых систем, может не описывать на локальном уровне динамические процессы даже в тех неупорядоченных твердотельных средах, которые хорошо описываются этой моделью на макроскопическом уровне.

3. Локальная динамика реальных неупорядоченных твердотельных систем при низких температурах может согласовываться со стандартной моделью низкотемпературных стекол в одних точках и одновременно не согласовываться с этой моделью в других точках одного и того же макроскопически однородного неупорядоченного твердотельного вещества.

4. Физические причины нарушения предсказаний стандартной модели низкотемпературных стекол при описании локальной спектральной динамики в неупорядоченных твердотельных молекулярных веществах, наблюдаемой с помощью одиночных флуоресцентных молекул, внедряемых в изучаемое вещество в качестве спектрального нанозонда, продолжают оставаться неясными.

5. Вопрос о причинах универсальности динамических свойств неупорядоченных твердотельных тел, обусловленных процессами внутренней динамики, остается открытым и подлежит дальнейшим исследованиям.

\section{Финансирование работы}

Работа выполнена в рамках Госзадания.

\section{Конфоликт интересов}

У автора нет конфликта интересов. 


\section{Список литературы}

[1] Zeller R.C., Pohl R.O. // Phys. Rev. B. 1971. V. 4. P. 2029.

[2] Amorphous Solids: Low Temperature Properties / Ed. by Phillips W.A.. Berlin, Heidelberg: Springer Verlag, 1981.

[3] Anderson P.W. // Science. 1995. V. 267. P. 1609. doi 10.1126/science.267.5204.1609-a

[4] Berthier L., Giroli B., Bouchaud J.-P., Cipeletti L., van Saarloos $W$. Dynamical Heterogeneities in Glasses, Colloids and Granular Media, Oxford: Oxford University Press, 2011. doi 10.1093/ACPROF:OSO/9780199691470.001.0001

[5] Wolynes P.G., Lubchenko V. Structural Glasses and Supercooled Liquids: Theory, Experiment and Applications, Hoboken, NJ: John Wiley and Sons, 2012.

[6] Phillips W.A. // J. Low Temp. Phys. 1972. V. 7. P. 351.

[7] Anderson P.W., Halperin B.I., Varma C.M. // Philos. Mag. 1972. V. 25. P. 1.

[8] Schechter M., Nalbach P., Burin A.L. // New J. Phys. 2018. V. 20. P. 063048. doi 10.1088/1367-2630/aac930

[9] Vorobyov V.V., Kazakov A.Yu., Soshenko V.V., Korneev A.A., Shalaginov M.Y., Bolshedvorskii S.V., Sorokin V.N., Divochiy A.V., Vakhtomin Yu.B., Smirnov K.V., Voronov B.M., Shalaev V.M., Akimov A.V., Goltsman G.N. // Opt. Matter. Express. 2017. V. 7. P. 513. doi 10.1364/OME.7.000513

[10] Single-Molecule Optical Detection, Imaging and Spectroscopy / Ed. by Basché Th., Moerner W.E., Orrit M., Wild U.P., Weinheim, NY., 1996.

[11] Moerner W.E., Shechtman Y., Wang Q. // Faraday Discussions. 2015. V. 184. P. 9. doi 10.1039/C5FD00149H

[12] Ambrose W.P., Moerner W.E. // Nature. 1991. V. 349. P. 225. doi 10.1038/349225A0

[13] Tittel J., Kettner R., Basché Th., Bräuchle C., Quante H., Müllen K. // J. Lumin. 1995. V. 64. P. 1. doi 10.1016/00222313(95)00002-8

[14] Boiron A.-M., Tamarat Ph., Lounis B., Brown R., Orrit M. // Chem. Phys. 1999. V. 247. P. 119. doi 10.1016/S03010104(99)00140-8

[15] Vainer Yu.G., Naumov A.V., Bauer M., Kador L. // J. Lumin. 2007. V. 127. P. 213. doi 10.1016/J.LUMIN.2007.02.026

[16] Vainer Yu.G., Naumov A.V., Kador L. // Phys. Rev. B. 2008. V. 77. P. 224202. doi 10.1103/PHYSREVB.77.224202

[17] Eremchev Yu., Vainer Yu.G., Naumov A.V., Kador L. // Phys. Chem. Chem. Phys. 2011. V. 13. P. 1843. doi $10.1039 / \mathrm{C} 1 \mathrm{CP} 90002 \mathrm{~A}$

[18] Naumov A.V., Vainer Yu.G., Kador L. // Phys. Rev. Lett. 2007. V. 98. P. 145501. doi 10.1103/PHYSREVLETT.98.145501

[19] Sobolev Ya.I., Naumov A.V., Vainer Yu.G., Kador L. // J. Chem. Phys. 2014. V. 140. P. 204907. doi 063/1.4879062

[20] Vainer Yu.G., Sobolev Ya.I., Naumov A.V., Osad'ko I.S., Kador L. // Faraday Discussions, 2015. V. 184. P. 237. doi 10.1039/C5FD00055F

[21] Lisenfeld J, Grabovskij G.J, Müller C., Cole J.H., Weiss G., Ustinov A.U. // Nature Commun. 2015. V. 6. N 6182. doi 10.1038/NCOMMS7182

[22] Geva E., Skinner J.L. // J. Phys. Chem. B. 1998. V. 109. P. 4920. doi: 10.1063/1.477103 Commun. 2015. V. 6. N 6182. doi 10.1038/NCOMMS7182

[23] Asban O., Amir I.A., Imry Y., Schechtert M. // Phys. Rew. B. 2017. V. 95. P. 144207. doi 10.1103/PHYSREVB.95.144207 